Aliment Pharmacol Ther. 2020 February ; 51(4): 435-445. doi:10.1111/apt.15577.

\title{
Prevalence of endoscopic improvement and endoscopic remission according to patient reported outcomes in ulcerative colitis
}

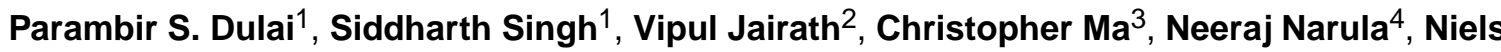 \\ Vande Casteele $^{1}$, Laurent Peyrin-Biroulet ${ }^{5}$, Severine Vermeire ${ }^{6}$, Geert D’Haens ${ }^{7}$, Brian G. \\ Feagan $^{2}$, William J. Sandborn ${ }^{1}$ \\ 1. University of California San Diego, La Jolla, CA, USA 2.University of Western Ontario, London, \\ Ontario, Canada ${ }^{3}$ University of Calgary, Calgary, Alberta, Canada ${ }^{4}$ McMaster University, \\ Hamilton, Ontario, Canada ${ }^{5}$ Nancy University Hospital, Nancy, France ${ }^{6}$.University Hospitals \\ Leuven, Leuven, Belgium ${ }^{7}$.Academic Medical Center, Amsterdam, The Netherlands
}

\section{Abstract}

Background: We quantified the prevalence of endoscopic improvement and remission amongst ulcerative colitis patients with various permutations of patient reported outcomes.

\begin{abstract}
Methods: Individual participant data from active intervention and placebo arms of clinical trials of infliximab, golimumab, vedolizumab, and tofacitinib were pooled to estimate the prevalence of endoscopic improvement (Mayo endoscopic sub-score [MES] 0 or 1) and remission (MES 0) scores with various permutations of the rectal bleeding sub-score (RBS) and stool frequency subscore (SFS) of the Mayo score, following induction (6-8 weeks) and maintenance (30-54 weeks) therapy. Subgroup analyses were performed by year of publication and centrally read endoscopy scoring.
\end{abstract}

Results: Data from 2,586 trial participants were analyzed. Using locally scored endoscopy, the prevalence of endoscopic improvement and remission was highest among participants with a RBS $0+$ SFS 0 post induction (MES 0/1: 81\%, [95\% CI 78-84]; MES 0:29\% [26-33]) and during maintenance (MES 0/1: 91\% [87-93]; MES 0: 57\% [52-62]). Prevalence estimates were lower for more recently performed trials $(\mathrm{p}<0.01)$. In comparison to locally scored endoscopy, when using central endoscopy scoring the prevalence of endoscopic improvement and remission were lower post-induction (MES 0/1 57\% [50-64], p<0.001; MES 0 15\% [11-21], p=0.09) and during maintenance (MES 0/1 74\% [67-81], $\mathrm{p}=0.001$; MES $031 \%$ [24-38], $\mathrm{p}=0.001$ ) for participants achieving a RBS $0+$ SFS 0.

\footnotetext{
Corresponding Author:Parambir S. Dulai, Assistant Professor of Medicine, Division of Gastroenterology, University of California San Diego, 9452 Medical Center Dr., La Jolla, CA 92093, pdulai@ ucsd.edu, Phone: 858-657-5284, Fax: 858-657-7259.

Author Contributions: PSD, SS, and WJS created the study concept and design. PSD, NVC, NN, and SS acquired the data. PSD and SS conducted the analyses and drafted the manuscript. All authors participated in the critical revision of the manuscript for important intellectual content. PSD provided study supervision.
} 
Conclusions: Approximately 8 out of 10 patients with normalization of rectal bleeding and stool frequency have improvement in endoscopic disease activity, whereas, approximately only half of these patients have endoscopic remission.

\section{Keywords}

patient reported outcomes; endoscopic remission; treatment target

\section{INTRODUCTION}

Patient reported outcomes are self-administered assessments of disease activity without interpretation by a health care provider. In ulcerative colitis, the most commonly used patient reported outcomes are the rectal bleeding sub-score (RBS) and stool frequency sub-score (SFS), of the Mayo score. ${ }^{1,2}$ Two recent systematic reviews which evaluated the operating properties of the RBS and SFS for non-invasive assessment of endoscopic inflammation, concluded that normalization of these assessments is strongly associated with the presence of endoscopic improvement (MES 0 or 1) which comprises both endoscopic remission (MES 0) and mildly active disease (MES 1). ${ }^{3,4}$ Notwithstanding that these reviews underscored the importance of patient reported outcome assessment in ulcerative colitis, several limitations remain in our understanding of the relationships between patient reported outcomes and endoscopy that warrant further investigation.

First, both prior reviews incorporated data from a limited sub-set of clinical trials and observational studies that carried a moderate-high risk of bias and they did not evaluate the association of symptoms specifically with achieving endoscopic remission (MES 0). Second, their analyses of the included clinical trial data did not consider the potential effects of the evolution in scoring friability for the Mayo endoscopic sub-score (MES). For example during the ACT trials that were conducted in the early 2000s, "mild" friability was scored as a MES 1, whereas in modern trials the presence of any friability is assigned a MES of 2. 5,6 Third, the lack of individual participant data from the studies analyzed precluded specific evaluation of all of the permutations of patient reported outcome-defined health states and their relationship to endoscopic disease activity. Fourth, relevant sub-group analyses of the data specific to the timing of treatment initiation (post-induction versus maintenance) were not preformed. Finally, because centrally read endoscopy, in distinction to local reading by site investigators, has become a gold standard in recent years it is unclear if the previously reported estimates of association between patient reported outcomes and endoscopic remission or mildly active disease reported in these reviews remain valid.

Addressing these limitations is relevant to better understand how patient reported outcomes can be optimally incorporated into both clinical trial designs and routine practice alogrithms. ${ }^{7}$ Accordingly, to better quantify the relationship between patient reported outcome defined health states and endoscopic disease activity, we analyzed individual patient data from induction and maintenance trials that spanned a broad spectrum of approved treatment options for moderate to severely active ulcerative colitis. 


\section{METHODS}

\section{Study Design and Data Sources}

Our primary objective was to estimate the prevalence of endoscopic improvement (MES 0/1) and endoscopic remission (MES 0) for various patient reported outcome permutations postinduction or during maintenance therapy for ulcerative colitis patients treated with drugs currently approved for moderate to severely active ulcerative colitis. Accordingly, the study was not designed as a systematic review, but rather focused on obtaining relevant data available though open access data sharing platforms (YODA \#2017-2031) or from industrysponsored registration trials. Based upon these criteria we were able to utilize individual patient data from ulcerative colitis clinical trials of infliximab (ACT-1 and -2), ${ }^{8}$ golimumab (PURSUIT-SC), ${ }^{9,} 10$ vedolizumab (MLN02 and GEMINI-1), ${ }^{11,} 12$ and tofacitinib (OCTAVE-1, -2 and SUSTAIN). ${ }^{13}$ (Supplementary Table 1) Participants from included trials were included from both the active intervention and placebo arms of trials, irrespective of induction response status, and prevalence estimates for endoscopic improvement and remission are therefore for the combined population of patients receiving active intervention (i.e. biologic or tofacitinib) or placebo. All included trials required a baseline MES of 2/3 for eligibility, that was derived by local scoring in all trials except the tofacitinib studies where central endoscopy scoring was used. Post-induction endoscopic assessments were performed at week 6 for vedolizumab and golimumab trial programs, and week 8 for infliximab and tofacitinib trial programs. Maintenance therapy assessments were performed at week 30 for infliximab and weeks 52-54 for infliximab, vedolizumab, and tofacitinib trial programs.

\section{Patient Reported and Endoscopic Outcomes}

Patient reported outcomes - The two patient reported outcomes assessed were the RBS and SFS sub-components of the Mayo score. RB was scored as 0 (none), +1 (visible blood with stool less than half the time), +2 (visible blood with stool half of the time or more), or +3 (passing blood alone). SF was scored as 0 (normal number of stools), +1 (1-2 stools/day more than normal), +2 (3-4 stools/day more than normal), or +3 ( $\geq 5$ stools/day more than normal). Resolution of rectal bleeding (RBS 0) has been observed to be most closely associated with achievement of treatment target of inactive or minimally endoscopically active disease and therefore the patient reported outcome permutations were anchored around RBS with different possible permutations of SFS. ${ }^{14}$ The pre-defined patient reported outcome permutations of interest were: RBS $0+$ SFS 0 , RBS $0+$ SFS 0/1, RBS $0+$ SFS $2 / 3$, and RBS $2 / 3+$ SFS $2 / 3$. These permutations were assessed separately postinduction (6-8 weeks) and during maintenance (30-54 weeks).

Endoscopy - The MES is a 4-point scale (0-3), which incorporates mucosal erythema, vascular pattern, friability, erosions, spontaneous bleeding and ulcerations. The use of friability in the scoring definition of MES changed over the course of the included trials and therefore secondary analyses were also performed using a more stringent definition of endoscopic remission defined as a MES 0. Endoscopic improvement was defined as a MES of 0 or 1 , in keeping with primary trial definitions and STRIDE recommendations. ${ }^{1}$ Endoscopic remission was defined as MES of 0. For the OCTAVE trials, both local and 
central endoscopy scoring were available and when pooling data across studies the local scores were used.

\section{Statistical Analyses}

Data were pooled across studies using Mantel-Haenszel fixed-effects model (in the absence of conceptual heterogeneity) and presented as prevalence of endoscopic remission (MES 0), mildly active disease (MES 1) and endoscopic improvement (MES 0 or 1), using forest plots with studies arranged by year of publication. Statistical assessment of heterogeneity in prevalence estimates for patient reported outcome permutations across studies was performed using the inconsistency index $\left(\mathrm{I}^{2}\right)$, which estimates what proportion of total variation across studies was due to heterogeneity rather than chance; a value of $>50 \%$ is suggestive of considerable heterogeneity. A comparison of prevalence estimates across the specified patient reported outcome permutations for local versus central endoscopy scores was done using local and central reads from OCTAVE $-1,-2$, and SUSTAIN trials. For scenarios with zero events, we applied a continuity correction of 0.5 added to each cell, based on the Cochrane handbook.

\section{RESULTS}

\section{Prevalence of endoscopic improvement (MES 0/1) by RBS and SFS permutations}

Individual patient data for 2,586 and 862 participants were available post-induction (6-8 weeks) and during maintenance (weeks 30-54) for estimation of the endoscopic outcomes for the pre-defined permutations. (Figures 1 and 2) The highest prevalence of endoscopic improvement post-induction $(81 \%, 95 \%$ CI $78-84 \%)$, and during maintenance $(91 \%, 95 \%$ CI 87-93\%), was observed for participants with a RBS $0+$ SFS 0 . In contrast the lowest prevalence of endoscopic improvement post-induction (10\%, 95\% CI 8-13\%), and during maintenance $(15 \%, 95 \%$ CI $8-25 \%)$, was observed for participants with a RBS $2 / 3+$ SFS $2 / 3$. (Figures $1 \mathrm{~A}$ and $2 \mathrm{~A}$ )

\section{Prevalence of endoscopic remission (MES 0) by RBS and SFS permutations}

The prevalence of endoscopic remission specifically among participants with a RBS 0 was $20 \%$ (95\% CI 18-22\%) post-induction, and 46\% (95\% CI 42-50\%) during maintenance. The prevalence of endoscopic remission among participants with a RBS $0+$ SFS 0 was $29 \%$ (95\% CI 26-33\%) post-induction, and 57\% (95\% CI 52-62\%) during maintenance.

Participants with a RBS $2 / 3+$ SFS $2 / 3$ had prevalence estimates for endoscopic remission of $<1 \%$ post-induction and $<5 \%$ during maintenance. (Figures $1 \mathrm{~B}$ and $2 \mathrm{~B}$ ).

\section{Heterogeneity in prevalence amongst trials}

Substantial heterogeneity was observed in prevalence estimates of endoscopic improvement post-induction and during maintenance amongst most permutations $\left(\mathrm{I}^{2}>50 \%\right)$. Prevalence estimates for MES 0/1 were consistently lower for recent trials (p-value for time-trend, $<0.01$ ). (Figures 1 and 2) This heterogeneity across studies was driven predominantly by heterogeneity in the prevalence of MES 0 for the RBS $0+$ SFS 0 (post-induction: $\mathrm{I}^{2}=82 \%$; maintenance: $\mathrm{I}^{2}=84 \%$ ) and RBS $0+$ SFS $0 / 1$ (post-induction: $\mathrm{I}^{2}=92 \%$; maintenance: $\mathrm{I}^{2}=86 \%$ ) permutations, and for MES 1 for the RBS $0+$ SFS 2/3 (post-induction: $\mathrm{I}^{2}=70 \%$; 
maintenance: $\mathrm{I}^{2}=51 \%$ ), and RBS $2 / 3+$ SFS $2 / 3$ (post-induction: $\mathrm{I}^{2}=52 \%$; maintenance: $\mathrm{I}^{2}=27 \%$ ) permutations. (Figures 1 and 2 )

\section{Effects of central reading of endoscopy}

Prevalence estimates for MES 0/1 and MES 0 and 1 separately were consistently lower with central scoring as compared to local scoring in the tofacitinib trials. (Tables 1 and 2) Postinduction, for participants with a RBS $0+$ SFS 0 the prevalence of MES $0 / 1$ was $77 \%$ (95\% CI 70-82\%) based upon the use of local endoscopy scores and 57\% (95\% CI 50-64\%) using central endoscopy scores ( $\mathrm{p}$-value for difference between estimates $<0.001$ ). The prevalence of MES 0 specifically in these participants was $22 \%$ (95\% CI 17-28\%) with local endoscopy scores and 15\% (95\% CI 11-21\%) with central endoscopy scores ( $\mathrm{p}=0.09)$. (Table 1) During maintenance therapy for participants with a RBS $0+$ SFS 0 the prevalence of MES 0/1 was $89 \%$ (95\% CI 83-93\%) based upon local reading and 74\% (95\% CI 67-81\%) for central reading $(\mathrm{p}=0.001)$. The prevalence of MES 0 specifically in these participants was $49 \%$ (95\% CI 41-57\%) for local reading and 31\% (95\% CI 24-38\%) for central reading $(\mathrm{p}=0.001)$. (Table 2)

\section{DISCUSSION}

The RBS and SFS are the cardinal patient reported outcomes used for assessment of disease activity in ulcerative colitis. Consequently, clinicians use them to assess response to induction therapy and monitor disease activity during maintenance. Their importance in management has been recognized in the consensus guidelines that identify resolution of rectal bleeding and normalization of stool frequency as treatment targets. ${ }^{1}$ Prior work has suggested that the majority of patients with complete resolution of rectal bleeding and normalization of stool frequency achieve endoscopic improvement (MES 0/1), ${ }^{3,4}$ however, these studies did not evaluate several important factors that may influence endoscopic disease activity including classification of endoscopic remission (MES 0) versus improvement (MES 0 or 1), timing of assessment (post-induction versus maintenance), evolution in scoring conventions regarding friability, or the influences of central scoring of endoscopy. In a pooled analysis of individual participant data for over 2,500 participants from pivotal ulcerative colitis clinical trial programs we have made several important observations, which extend prior work in this arena.

First, among participants with a RBS $0+$ SFS 0 , the traditional definition of mucosal healing which is now termed endoscopic improvement (MES 0/1), was observed in $81 \%$ of participants post-induction and in $91 \%$ during maintenance therapy. However, the modern definition of endoscopic remission (MES 0) was present in only $29 \%$ of these patients at the end of induction therapy and $57 \%$ during maintenance. With evolving treat-to-target strategies, these patients in complete clinical remission (RBS $0+$ SFS 0) but with mild endoscopic inflammation (MES 1) may potentially require treatment adjustment and/or transitions in therapy. The transitions in prevalence estimates over time, with higher prevalence estimates for MES 0/1 and MES 0 and lower prevalence estimates for MES 1 between induction and maintenance, would suggest that a transition from MES 1 to MES 0 is feasible over time simply with continued treatment exposure, thereby making it unclear if 
providers truly need to adjust therapy for mild activity (MES 1) post-induction or if they should simply wait and repeat endoscopic assessment to see if the patient has transitioned to full endoscopic remission (MES 0). In the ACT-1 and -2 clinical trials of infliximab in moderate-severe ulcerative colitis no significant difference was observed between patients achieving clinical remission + MES 0 versus those achieving clinical remission + MES 1 at week 8 , for the outcomes of symptomatic remission, corticosteroid-free remission, sustained mucosal healing, or need for colectomy over 1 year. ${ }^{15}$ In contrast, other retrospective cohort studies have suggested a lower risk of colectomy and clinical relapse in patients achieving MES 0 vs. 1, without consideration for the timing of assessment or achievement of this outcome. ${ }^{16-19}$ Recent evidence has emerged that cumulative burden of inflammation in ulcerative colitis is predictive of future risk for colorectal neoplasia, suggesting that treatment of persistent mild inflammation (MES 1) would be of importance. ${ }^{20}$ However, consideration will need to be given to the incremental value of transitioning from a MES 1 to MES 0 in the absence of clinical symptoms with regards to reductions in disease-related risks because, as we observed, this is a substantial proportion of the population in whom this issue would be directly applicable, treatment adjustments or transitions in therapy are not without risk or cost, some patients may naturally transition from MES 1 to MES 0 with continued treatment exposure, and high quality evidence supporting treatment adjustment for MES 1 are lacking.

Second, we observed that estimates for MES 0/1 and MES 0 increase over time for all of the patient reported outcome permutations. Rates were highest at the end of maintenance studies and relatively lower at the end of induction. While this observation is undoubtably partially accounted for by selection of responder to drug therapy as an eligibility criterion for participation in maintenance trials, it also holds out the highly plausible possibility that extended periods of induction beyond the conventional 8 to 10 weeks might result in greater endoscopic remission rates and superior separation from placebo in these studies. It is noteworthy that the origin of the relatively short duration of induction trials was influenced by the observation that placebo rates tend to increase over time reaching an apex at approximately 16 weeks. Accordingly, selection of an early induction timepoint served to minimize placebo response rates and increased trial efficiency. However in the modern era the incorporation of endoscopic criteria into the definition of clinical remission and the use of central reading of endoscopy has tended to minimize this phenomenon such that placebo treated patients tend to have stable and low remission rates even with prolonged induction beyond 10 weeks. Thus, it is likely that future trials may employ total durations of 12 weeks, which will offer greater statistical efficiency and have the advantage of more closely reflecting clinical practice where agents are evaluated for approximately 3 months before alternative treatments are introduced.

Third, significant heterogeneity was observed across trials for MES 0/1 and this was predominately driven by heterogeneity in prevalence estimates for MES 0 across trials, particularly among participants achieving complete resolution of rectal bleeding. This is actually in contrast to our expected observation that heterogeneity across trials for MES 0/1 would be driven by heterogeneity in prevalence estimates for MES 1 due to evolutions in convention regarding scoring of friability. The reason for this heterogeneity in MES 0 across trials over time is unclear, but evolution in high definition endoscopy and video imaging may 
play an important role in the assessment of endoscopic scoring going forward. ${ }^{21-23}$ This is further emphasized by our observations that prevalence estimates for MES 0 and 1 or 0/1 were consistently lower when using central endoscopy scoring as compared to local endoscopy scoring, and the prevalence of MES 0 among participants achieving a RBS $0+$ SFS 0 when using central endoscopy scoring was estimated to be $15 \%$ post-induction and $31 \%$ during maintenance. These finding are consistent with previous observations that local reading tends to overestimate endoscopic remission and response rates relative to those observed with central reading. Although the reason for this difference is poorly understand it may be due in part to the circumstance whereby the local reader is aware of the patient reported outcomes and is influenced by them during the scoring process.

Our study has several strengths such as the inclusion of trials spanning a wide spectrum of available treatment options for moderate to severely active ulcerative colitis, the use of pooled individual participant data derived from over 2,500 participants, and evaluation of the effects of patient reported outcome assessment timing and central versus local endoscopy scoring on the estimates. Some limitations warrant consideration. We were not able to account for variations in patient reported outcome collection techniques over time and whether these may have influenced observed heterogeneity in prevalence estimates for endoscopic remission. We did not have access to original endoscopic operative reports and therefore could not assess the number of participants with a MES of 1 on the basis of friability which could be helpful in understanding how the evolution friability scoring may have affected the prevalence estimates. These were post-hoc analyses of trial data and caution should be taken in their interpretation. Finally, one notable limitation is that the Mayo endoscopic sub-score does not accurately reflect partial improvements in endoscopic activity, and evolutions in endoscopic scoring indices such as the ulcerative endoscopic index of severity (UCEIS) may help to better define definitions for endoscopic remission which are readily achievable in the majority of patients achieving complete resolution of rectal bleeding and normalization of stool frequency and are significantly associated with reductions in disease-related complications. ${ }^{24-27}$

In summary, nearly $80 \%$ of participants from pivotal ulcerative colitis clinical trial programs who achieved a RBS $0+$ SFS 0 post-induction or during maintenance also achieved endoscopic improvement when defined as a MES 0 or 1 . However, when using more modern day definitions for endoscopic remission (MES 0), only half of patients with a RBS $0+$ SFS 0 have endoscopic remission during maintenance. These findings highlight a disconnect between normalization of symptoms and achievement of full endoscopic remission, the need for monitoring both symptoms and endoscopic lesions in order to achieve a full disease control in ulcerative colitis patients, and an emphasis being placed on the generation of high quality evidence to support a transition in definitions towards MES 0 for remission.

\section{Supplementary Material}

Refer to Web version on PubMed Central for supplementary material. 


\section{Acknowledgement:}

Parambir S. Dulai is supported by an American Gastroenterology Association Research Scholar Award. Siddharth Singh is supported by an American College of Gastroenterology Junior Faculty Development Award \#144271, Crohn's and Colitis Foundation Career Development Award \#404614, and the National Institute of Diabetes and Digestive and Kidney Diseases K23DK117058. The content is solely the responsibility of the authors and does not necessarily represent the official views of the National Institutes of Health. Neeraj Narula is supported by a McMaster University Department of Medicine Internal Career Award.

Data Source Acknowledgement: This study, carried out under YODA Project \#2017-2031, used data obtained from the Yale University Open Data Access Project, which has an agreement with JANSSEN RESEARCH \& DEVELOPMENT, L.L.C.. The interpretation and reporting of research using this data are solely the responsibility of the authors and does not necessarily represent the official views of the Yale University Open Data Access Project or JANSSEN RESEARCH \& DEVELOPMENT, L.L.C.

Conflicts of Interest: PSD has received research support from Takeda, Pfizer, Abbvie, Janssen, Polymedco, ALPCO, Buhlmann, and consulting fees from Takeda, Pfizer, Abbvie and Janssen. SS has received research grants from AbbVie, and consulting fees from AbbVie, Takeda, Pfizer, AMAG Pharmaceuticals. $V J$ has received has received consulting fees from AbbVie, Eli Lilly, GlaxoSmithKline, Arena pharmaceuticals, Genetech, Pendopharm, Sandoz, Merck, Takeda, Janssen, Robarts Clinical Trials, Topivert, Celltrion; speaker's fees from Takeda, Janssen, Shire, Ferring, Abbvie, Pfizer. $C M$ has served as a consultant for Robarts Clinical Trials, Inc., advisory board for Janssen, AbbVie, speakers fees from Janssen, Pfizer. NNhas received honoraria from Janssen, Abbvie, Takeda, Pfizer, Merck, and Ferring. MM has received speaker and consultant fees for Takeda, Abbvie, Janssen, and Pfizer. $N V C$ has received research support from R-Biopharm and Takeda and consulting fees from Boehringer Ingelheim, Janssen, Pfizer, Progenity, Prometheus and Takeda, outside of the submitted work. $L B P$ has recieved honoraria from AbbVie, Janssen, Genentech, Ferring, from Tillots, Pharmacosmos, Celltrion, Takeda, Boerhinger Ingelheim, Pfizer, Index Pharmaceuticals, Sandoz, Celgene, Biogen, Samsung Bioepis, Alma, Sterna, Nestle, Enterome, Allergan, MSD, Roche, Arena, Gilead, Hikma, Amgen; grants from Abbvie, MSD, Takeda; stock options : CT-SCOUT. $S V$ has received grant support from AbbVie, MSD, Janssen and Takeda; received speaker fees from AbbVie, MSD, Takeda, Ferring, Dr. Falk Pharma, Hospira, Pfizer Inc and Tillots; and served as a consultant for AbbVie, MSD, Takeda, Ferring, Genentech/Roche, Shire, Pfizer Inc, Galapagos, Amgen, Gilead, Mundipharma, Hospira, Celgene, Second Genome, and Janssen. GDH has served as advisor for Abbvie, Ablynx, Amakem, AM Pharma, Avaxia, Biogen, Bristol Meiers Squibb, Boerhinger Ingelheim, Celgene, Celltrion, Cosmo, Covidien, Ferring, DrFALK Pharma, Engene, Galapagos, Gilead, Glaxo Smith Kline, Hospira, Immunic, Johnson and Johnson, Lycera, Medimetrics, Millenium/Takeda, Mitsubishi Pharma, Merck Sharp Dome, Mundipharma, Novonordisk, Pfizer, Prometheus laboratories/Nestle, Protagonist, Receptos, Robarts Clinical Trials, Salix, Sandoz, Setpoint, Shire, Teva, Tigenix, Tillotts, Topivert, Versant and Vifor and received speaker fees from Abbvie, Ferring, Johnson and Johnson, Merck Sharp Dome, Mundipharma, Norgine, Pfizer, Shire, Millenium/Takeda, Tillotts and Vifor. $B G F$ has received grant/research support from Millennium Pharmaceuticals, Merck, Tillotts Pharma, AbbVie, Novartis Pharmaceuticals, Centocor, Elan/Biogen, UCB Pharma, Bristol-Myers Squibb, Genentech, ActoGenix and Wyeth Pharmaceuticals; consulting fees from Millennium Pharmaceuticals, Merck, Centocor, Elan/Biogen, Janssen-Ortho, Teva Pharmaceuticals, Bristol-Myers Squibb, Celgene, UCB Pharma, AbbVie, AstraZeneca, Serono, Genentech, Tillotts Pharma, Unity Pharmaceuticals, Albireo Pharma, Given Imaging, Salix Pharmaceuticals, Novonordisk, GSK, ActoGenix, Prometheus Therapeutics and Diagnostics, Athersys, Axcan, Gilead, Pfizer, Shire, Wyeth, Zealand Pharma, Zyngenia, GiCare Pharma and Sigmoid Pharma; and speaker's bureau fees from UCB, AbbVie and $\mathrm{J} \& \mathrm{~J} / \mathrm{J} a n s s e n . W J S$ has received research grants from Atlantic Healthcare Limited, Amgen, Genentech, Gilead Sciences, Abbvie, Janssen, Takeda, Lilly, Celgene/Receptos; consulting fees from Abbvie, Allergan, Amgen, Arena Pharmaceuticals, Avexegen Therapeutics, BeiGene, Boehringer Ingelheim, Celgene, Celltrion, Conatus, Cosmo, Escalier Biosciences, Ferring, Forbion, Genentech, Gilead Sciences, Gossamer Bio, Incyte, Janssen, Kyowa Kirin Pharmaceutical Research, Landos Biopharma, Lilly, Oppilan Pharma, Otsuka, Prizer, Precision IBD, Progenity, Prometheus Laboratories, Reistone, Ritter Pharmaceuticals, Robarts Clinical Trials (owned by Health Academic Research Trust, HART), Series Therapeutics, Shire, Sienna Biopharmaceuticals, Sigmoid Biotechnologies, Sterna Biologicals, Sublimity Therapeutics, Takeda, Theravance Biopharma, Tigenix, Tillotts Pharma, UCB Pharma, Ventyx Biosciences, Vimalan Biosciences, Vivelix Pharmaceuticals; and stock or stock options from BeiGene, Escalier Biosciences, Gossamer Bio, Oppilan Pharma, Precision IBD, Progenity, Ritter Pharmaceuticals, Ventyx Biosciences, Vimalan Biosciences. Spouse: Opthotech-consultant, stock options; Progenity-consultant, stock; Oppilan Pharma-employee, stock options; Escalier Biosciences-employee, stock options; Precision IBD-employee, stock options; Ventyx Biosciences - employee, stock options; Vimalan Biosciences - employee, stock options.

\section{References}

1. Peyrin-Biroulet L, Sandborn W, Sands BE, et al. Selecting Therapeutic Targets in Inflammatory Bowel Disease (STRIDE): Determining Therapeutic Goals for Treat-to-Target. Am J Gastroenterol 2015;110:1324-38. [PubMed: 26303131] 
2. Jairath V, Khanna R, Zou GY, et al. Development of interim patient-reported outcome measures for the assessment of ulcerative colitis disease activity in clinical trials. Aliment Pharmacol Ther 2015;42:1200-10. [PubMed: 26388424]

3. Narula N, Alshahrani AA, Yuan Y, et al. Patient-Reported Outcomes and Endoscopic Appearance of Ulcerative Colitis-a Systematic Review and Meta-Analysis. Clin Gastroenterol Hepatol 2018.

4. Restellini S, Chao CY, Martel M, et al. Clinical Parameters Correlate With Endoscopic Activity of Ulcerative Colitis: A Systematic Review. Clin Gastroenterol Hepatol 2019;17:1265-1275 e8. [PubMed: 30583048]

5. Parsi MA, Lashner BA. Safety of infliximab: primum non nocere. The safety profile of infliximab in patients with Crohn's disease: the Mayo Clinic experience in 500 patients. Inflammatory Bowel Diseases 2004;10:486-7. [PubMed: 15475765]

6. Scherl EJ, Pruitt R, Gordon GL, et al. Safety and efficacy of a new $3.3 \mathrm{~g}$ b.i.d. tablet formulation in patients with mild-to-moderately-active ulcerative colitis: a multicenter, randomized, double-blind, placebo-controlled study. Am J Gastroenterol 2009;104:1452-9. [PubMed: 19491859]

7. Dulai PS, Peyrin-Biroulet L. Integrating Patient-Reported Outcomes Into Treat-to-Target Monitoring Algorithms. Clin Gastroenterol Hepatol 2018.

8. Rutgeerts P, Sandborn WJ, Feagan BG, et al. Infliximab for induction and maintenance therapy for ulcerative colitis. N Engl J Med 2005;353:2462-76. [PubMed: 16339095]

9. Sandborn WJ, Feagan BG, Marano C, et al. Subcutaneous golimumab induces clinical response and remission in patients with moderate-to-severe ulcerative colitis. Gastroenterology 2014;146:85-95; quiz e14-5. [PubMed: 23735746]

10. Sandborn WJ, Feagan BG, Marano C, et al. Subcutaneous golimumab maintains clinical response in patients with moderate-to-severe ulcerative colitis. Gastroenterology 2014;146:96-109 e1. [PubMed: 23770005]

11. Feagan BG, Rutgeerts P, Sands BE, et al. Vedolizumab as induction and maintenance therapy for ulcerative colitis. N Engl J Med 2013;369:699-710. [PubMed: 23964932]

12. Feagan BG, Greenberg GR, Wild G, et al. Treatment of ulcerative colitis with a humanized antibody to the alpha4beta7 integrin. N Engl J Med 2005;352:2499-507. [PubMed: 15958805]

13. Sandborn WJ, Su C, Sands BE, et al. Tofacitinib as Induction and Maintenance Therapy for Ulcerative Colitis. N Engl J Med 2017;376:1723-1736. [PubMed: 28467869]

14. Colombel JF, Keir ME, Scherl A, et al. Discrepancies between patient-reported outcomes, and endoscopic and histological appearance in UC. Gut 2017;66:2063-2068. [PubMed: 27590995]

15. Colombel JF, Rutgeerts P, Reinisch W, et al. Early mucosal healing with infliximab is associated with improved long-term clinical outcomes in ulcerative colitis. Gastroenterology 2011;141:1194201. [PubMed: 21723220]

16. Boal Carvalho P, Dias de Castro F, Rosa B, et al. Mucosal Healing in Ulcerative Colitis--When Zero is Better. J Crohns Colitis 2016;10:20-5. [PubMed: 26438714]

17. Barreiro-de Acosta M, Vallejo N, de la Iglesia D, et al. Evaluation of the Risk of Relapse in Ulcerative Colitis According to the Degree of Mucosal Healing (Mayo 0 vs 1): A Longitudinal Cohort Study. J Crohns Colitis 2016;10:13-9. [PubMed: 26351390]

18. Nakarai A, Kato J, Hiraoka S, et al. Prognosis of ulcerative colitis differs between patients with complete and partial mucosal healing, which can be predicted from the platelet count. World J Gastroenterol 2014;20:18367-74.

19. Manginot C, Baumann C, Peyrin-Biroulet L. An endoscopic Mayo score of 0 is associated with a lower risk of colectomy than a score of 1 in ulcerative colitis. Gut 2015;64:1181-2.

20. Choi CR, Al Bakir I, Ding NJ, et al. Cumulative burden of inflammation predicts colorectal neoplasia risk in ulcerative colitis: a large single-centre study. Gut 2017.

21. Bossuyt P, Vermeire S, Bisschops R. Scoring endoscopic disease activity in IBD: artificial intelligence sees more and better than we do. Gut 2019.

22. Ozawa T, Ishihara S, Fujishiro M, et al. Novel computer-assisted diagnosis system for endoscopic disease activity in patients with ulcerative colitis. Gastrointest Endosc 2019;89:416-421.e1. [PubMed: 30367878] 
23. Stidham RW, Liu W, Bishu S, et al. Performance of a Deep Learning Model vs Human Reviewers in Grading Endoscopic Disease Severity of Patients With Ulcerative Colitis. JAMA Netw Open 2019;2:e193963.

24. Travis SP, Schnell D, Feagan BG, et al. The Impact of Clinical Information on the Assessment of Endoscopic Activity: Characteristics of the Ulcerative Colitis Endoscopic Index Of Severity [UCEIS]. J Crohns Colitis 2015;9:607-16. [PubMed: 25956538]

25. Travis SP, Schnell D, Krzeski P, et al. Reliability and initial validation of the ulcerative colitis endoscopic index of severity. Gastroenterology 2013;145:987-95. [PubMed: 23891974]

26. Arai M, Naganuma M, Sugimoto S, et al. The Ulcerative Colitis Endoscopic Index of Severity is Useful to Predict Medium-to Long-Term Prognosis in Ulcerative Colitis Patients with Clinical Remission. J Crohns Colitis 2016;10:1303-1309. [PubMed: 27194529]

27. Ikeya K, Hanai H, Sugimoto K, et al. The Ulcerative Colitis Endoscopic Index of Severity More Accurately Reflects Clinical Outcomes and Long-term Prognosis than the Mayo Endoscopic Score. J Crohns Colitis 2016;10:286-95. [PubMed: 26581895] 
Patient-reported Outcomes

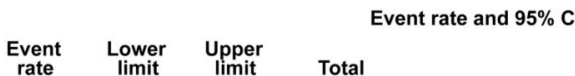

Study name, by year $\begin{gathered}\text { Event } \\ \text { rate }\end{gathered} \quad \begin{aligned} & \text { Lower } \\ & \text { limit }\end{aligned} \quad \begin{aligned} & \text { Upper } \\ & \text { limit }\end{aligned} \quad$ Total

of publication

2005, ACT-1

2005, ACT-2

2005, MLN02

2013, GEMINI 1

2014, PURSUIT-SC

2017, OCTAVE

0.775
0.749
0.735

$\begin{array}{ll}0.775 & 0.714 \\ 0.749 & 0.685\end{array}$

$0.735 \quad 0.639$

$0.601 \quad 0.55$

$\begin{array}{ll}0.667 & 0.624 \\ 0.572 & 0.533\end{array}$

RBS 0

$\mathbf{0 . 6 4 3} \quad 0.622$

2005, ACT-1

2005, ACT-2

2005, MLN02

2013, GEMSUIT-SC

$\begin{array}{ll}0.957 & 0.874\end{array}$

$0.952 \quad 0.829$

$0.947 \quad 0.813$

$\begin{array}{ll}0.769 & 0.693 \\ 0.869 & 0.800\end{array}$

2017, OCTAVE

0.869
0.765

RBS 0, SFS 0

2005, ACT-1

2005, ACT-2

2005, MLNO2

2013, GEMNITSC

2017, PCTAVE

RBS 0, SFS 0/1

2005, ACT-1

2005, ACT-2

2005, MLNO2

2013, GEMIN 1 -SC 2014, PURSUIT-S
2017, OCTAVE

0.812

0.701

0.827
0.804

0.804
0.813

0.813
0.649

$0.707-229 / 381$

$0.610-326 / 489$

$0.6641299 / 2006$

$0.986 \quad 66 / 69$

$0.988 \quad 40 / 42$

$0.987 \quad 36 / 38$

$0.831 \quad 110 / 143$

$0.917-113 / 130$

$150 / 196$

$\begin{array}{ll}0.777 & 0.843\end{array}$

515 / 618

$\begin{array}{llll}0.877 & 0.815 & 0.920 & 135 / 154\end{array}$

$\begin{array}{llll}0.877 & 0.815 & 0.920 & 13 / 154 \\ 0.848 & 0.781 & 0.897 & 128 / 151 \\ 0.729 & 0.670 & 0.927 & 65 / 75\end{array}$

$\begin{array}{llll}0.867 & 0.770 & 0.927 & 65 / 75 \\ 0.729 & 0.673 & 0.778 & 199 / 273\end{array}$

$\begin{array}{llll}0.729 & 0.673 & 0.927 & 65 / 75 \\ 0.780 & 0.734 & 0.821 & 270 / 346\end{array}$

$\begin{array}{llll}0.684 & 0.641 & 0.725 & 323 / 472\end{array}$

$270 / 346$
$323 / 472$

$\begin{array}{llll}0.753 & 0.730 & 0.775 & 1120 / 1471\end{array}$

0.527

0.462

0.304

0.278

0.392

0.396

0.332

0.153
0.202

0.202
0.315

0.315
0.168

0.654
0.597

0.597
0.515

0.369

0.474
0.300

RBS 0 , SFS $2 / 3$

0.345

0.305

2005, ACT-1

2005, ACT-2

2005, MLNO2

2013, GEMINI 1

2014, PURSUIT-SC 2017, OCTAVE

0.185

0.189

0.107

0.061
0.071

0.079

0.093

0.069

0.034
0.039

0.387

$29 / 55$

$24 / 52$

$7 / 23$

$30 / 108$

$56 / 143$

$35 / 154$

0.098

0.075

$\begin{array}{ll}0.375 & 5 / 27 \\ 0.347 & 7 / 37\end{array}$

$\begin{array}{ll}0.347 & 7 / 37 \\ 0.294 & 1 / 19\end{array}$

0.161

$0.108 \quad 11 / 179$

$0.128 \quad 10 / 140$

$53 / 580$

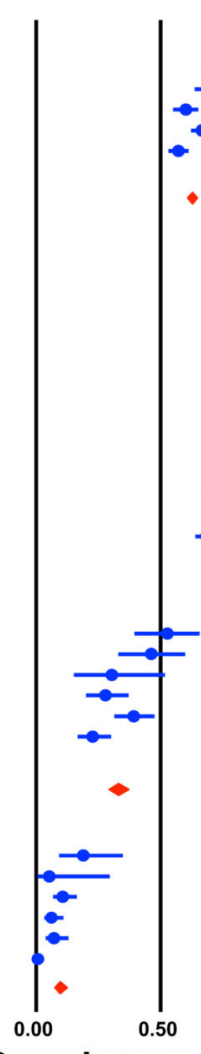

Prevalence

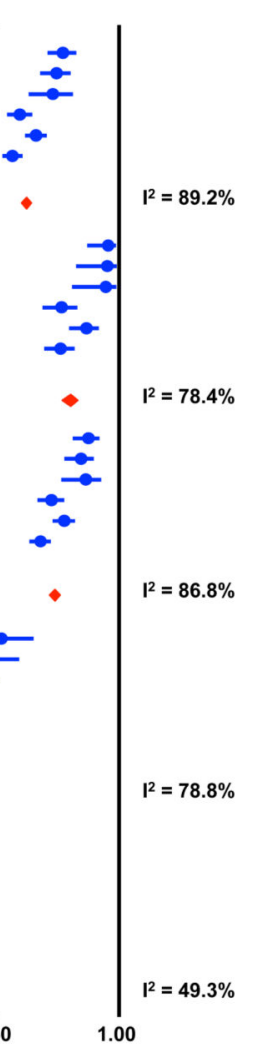


Patient-reported Outcomes Study name, by year of publication

2005, ACT-1

2005, MLN02

2013, GEMINI 1

2014, PURSUIT-SC

2017, OCTAVE

RBS 0

2005, ACT-2

2005, MLN02

2013, GEMINI 1

2014, PURSUIT-SC

2017, OCTAVE

RBS 0, SFS 0

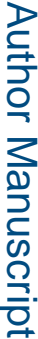

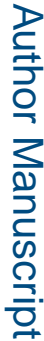

RBS 2/3, SFS 2/3
2005, ACT-1

2005, ACT-2

2005, MLN02

2013, GEMINI 1

2014, PURSUIT-SC

2017. OCTAVE

RBS 0, SFS 0/1

2005, ACT-1

2005, MLN02

2013, GEMINI 1

2014, PURSUIT-SC

2017, OCTAVE

RBS 0, SFS $2 / 3$

2005, ACT-1

2005, ACT- 1

2005, ACT-2

2005, MLN02

2013, GEMINI 1

2017. OCTAVE
Event rate and $95 \% \mathrm{Cl}$

Event Lower Upper
rate

0.316

0.305

0.286

0.139

0.168

0.147

limit

0.256
0.246

0.246

$0.108 \quad 0.178$

$0.137 \quad 0.203$

$\begin{array}{ll}0.121 & 0.177\end{array}$

0.199

0.181

0.217

0.449

0.500

0.368

0.203

0.300

0.219

0.337

0.353

0.232

$0.228 \quad 0.384$

$0.167 \quad 0.283$

0.294

0.258

0.332

0.403

0.371
0.373

0.373

0.172

0.211

0.328

$\begin{array}{ll}0.298 & 0.482\end{array}$

$0.298 \quad 0.451$

$0.272 \quad 0.487$

$\begin{array}{ll}0.132 & 0.222\end{array}$

$\begin{array}{ll}0.171 & 0.257 \\ 0.148 & 0.217\end{array}$

$0.148 \quad 0.217$

0.247

0.225

0.271

0.073

0.115

0.021

0.056

0.063

0.028

$0.028 \quad 0.178$

0.234

$0.025 \quad 0.118$

$0.033 \quad 0.117$

$0.022 \quad 0.092$

$66 / 209$

$62 / 203$

$28 / 98$

$53 / 381$

$82 / 489$

$92 / 626$

0.045

$0.046 \quad 0.089$

$383 / 2449$

0.064

$0.001 \quad 0.230$

$\begin{array}{ll}0.001 & 0.230 \\ 0.001 & 0.178\end{array}$

$0.001 \quad 0.178$

$\begin{array}{ll}0.002 & 0.298 \\ 0.001 & 0.039\end{array}$

$\begin{array}{ll}0.001 & 0.039 \\ 0.001 & 0.039\end{array}$

$\begin{array}{ll}0.001 & 0.039 \\ 0.000 & 0.054\end{array}$

$31 / 69$

$21 / 42$

$14 / 38$

$29 / 143$

$39 / 130$

$43 / 196$

$177 / 618$

$62 / 154$

$56 / 151$

$28 / 75$

$47 / 273$

$73 / 346$

$351 / 1471$

$4 / 55$

$6 / 52$

$0 / 23$

6/108

$9 / 143$

7 / 154

$0.003 \quad 0.020$

$32 / 535$

$0 / 27$

$0 / 37$

$0 / 19$

$1 / 178$

$1 / 179$
$0 / 140$

0.004

0.008

$2 / 580$

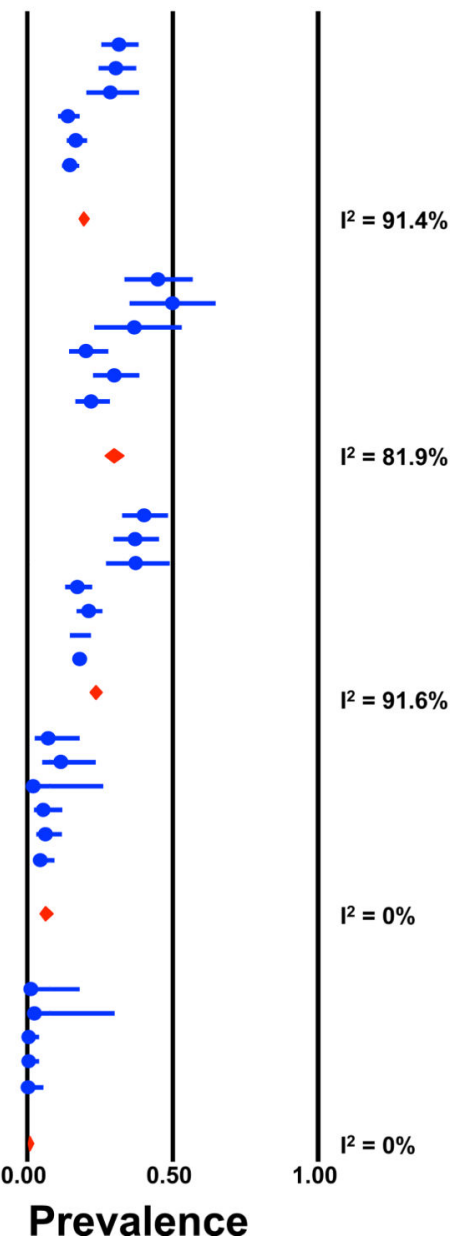




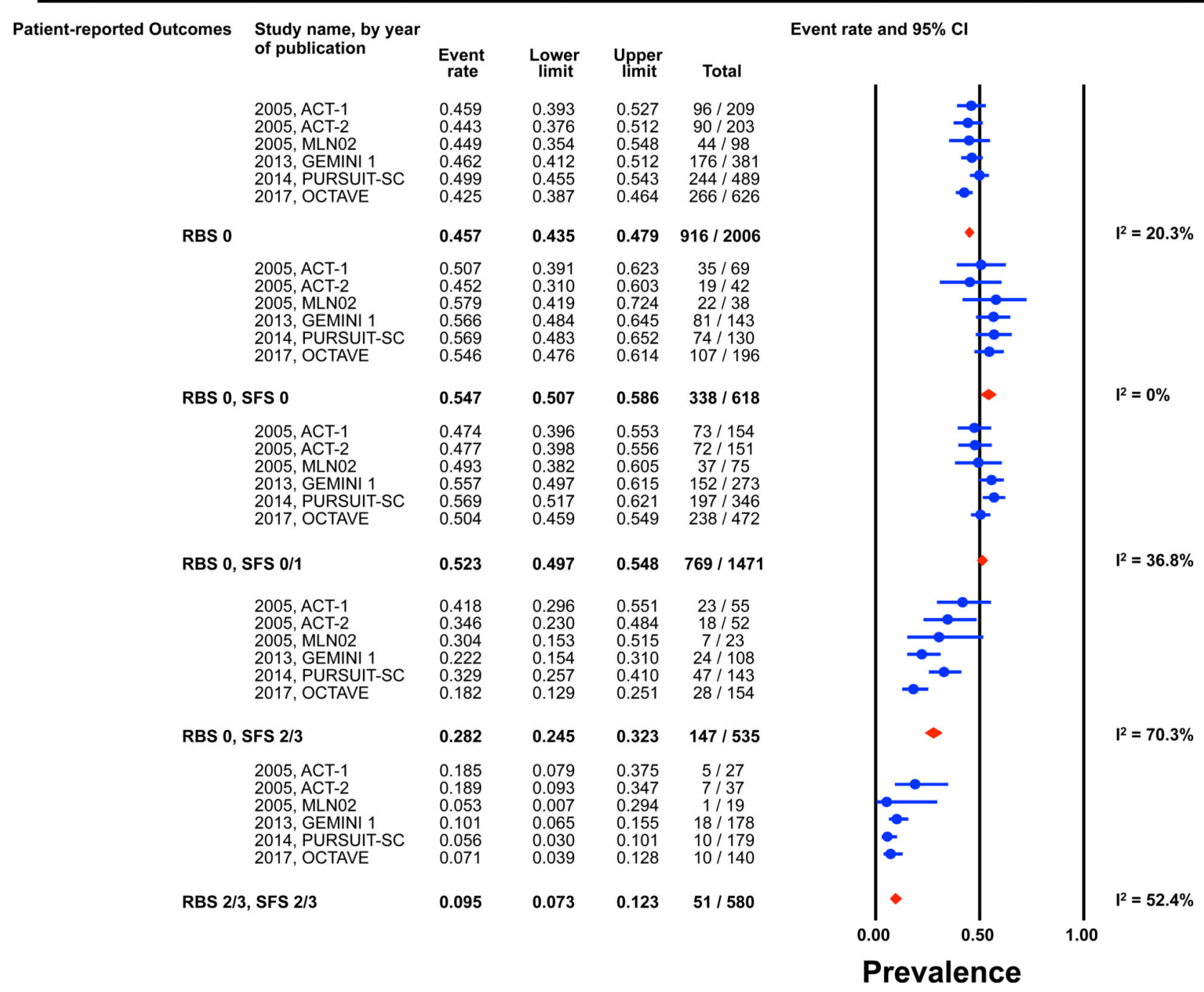

Figure 1: Pooled estimates from active intervention and placebo arms of ulcerative colitis clinical trials for prevalence of Mayo endoscopic scores post-induction by patient reported outcome permutations

RBS: rectal bleedings core; SFS: stool frequency score; CI: Confidence interval Prevalence estimates are for the combined population of patients receiving active intervention (ie biologic or tofacitinib) or placebo. Individual study level estimates cannot be used to compare prevalence estimates across studies between different biologics or tofacitinib. A: Endoscopic improvement (Mayo endoscopic score 0 or 1); B: Endoscopic remission (Mayo endoscopic scopre 0); C: Mild endoscopic activity (Mayo endoscopic score 1) 
Patient-reported Outcomes Study name, by year of publication

2005, ACT-1
2005, ACT-2
2013, GEMINI 1
2017, OCTAVE

RBS 0

2005, ACT-1
2005, ACT-2
2013, GEMINI 1
2017, OCTAVE

RBS 0, SFS 0

2005, ACT-1 2005, ACT-2 2013, GEMINI 1 2017, OCTAVE

RBS 0, SFS 0/1

2005, ACT-1
2005, ACT-2
2013, GEMINI 1
2017, OCTAVE

RBS 0 , SFS $2 / 3$

2005, ACT-1 2005, ACT-2 2013, GEMINI 1 2017, OCTAVE

RBS $2 / 3$, SFS $2 / 3$
Event

rate

0.892

0.802

0.829

0.800

0.820

0.963

0.977

0.899

0.894

0.907

0.928

0.920

0.867

0.856

0.876

0.643

0.419

0.500

0.333

0.446

0.095

0.167

0.222

0.016

0.145

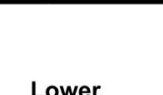

Lower

0.819

0.724

0.778

0.749

0.792

0.864

0.856

0.841

0.834

0.873

0.856

0.848

0.816

0.807

0.849

0.376

0.261

0.317

0.190

0.350

0.024

0.055

0.086

0.001

0.078

\section{Upper}

limit

0.938

0.861

0.871

0.843

0.846

0.991

0.997

0.937

0.934

0.932

0.965

0.959

0.906

0.894

0.899

0.843

0.596

0.683

0.516

0.546

0.311

0.409

0.465

0.206

0.254
Event rate and $95 \% \mathrm{Cl}$

Total

$99 / 111$

$105 / 131$

$209 / 252$

$224 / 280$

$637 / 774$

$52 / 54$

$43 / 44$

$142 / 158$

$135 / 151$

372 / 407

$90 / 97$

$92 / 100$

$196 / 226$

$214 / 250$

$592 / 673$

$9 / 14$

$13 / 31$

$13 / 26$

$10 / 30$

$45 / 101$

$2 / 21$

$3 / 18$

$4 / 18$

$0 / 31$

$9 / 88$

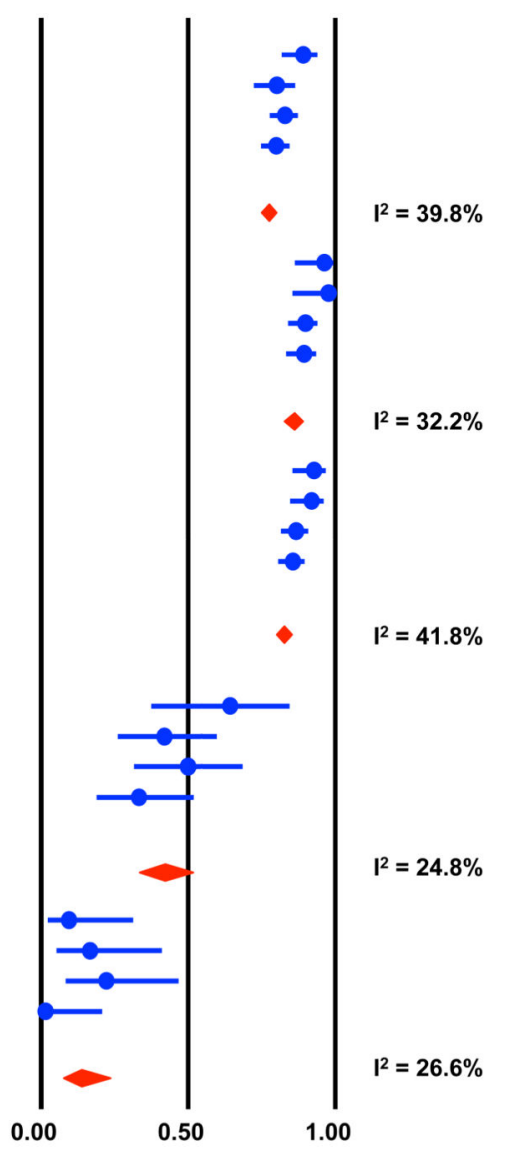

Prevalence 


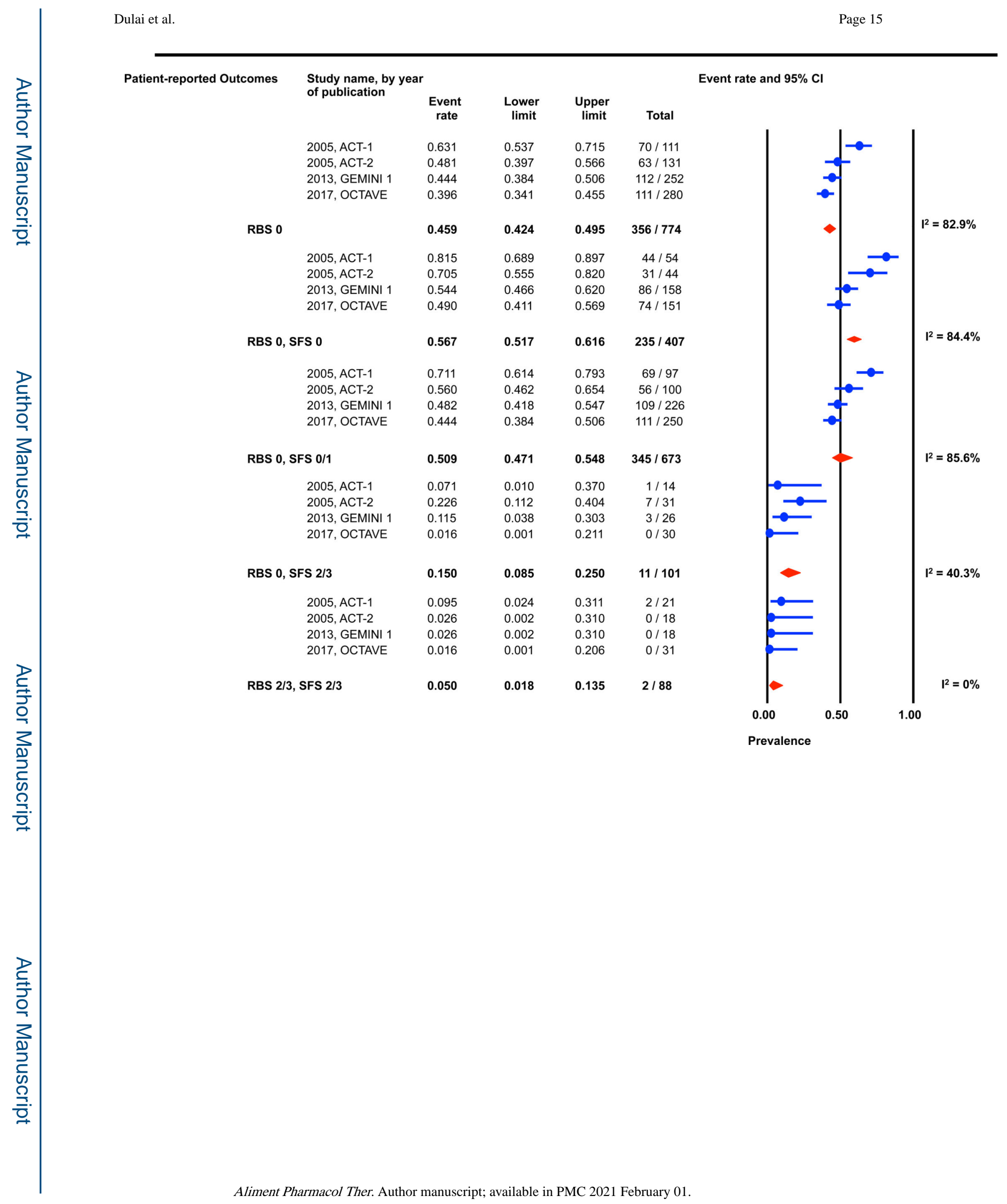


Patient-reported Outcomes Study name, by year of publication

\begin{tabular}{|c|c|c|c|c|}
\hline & rate & limit & limit & Total \\
\hline 2005, ACT-1 & 0.261 & 0.188 & 0.351 & $29 / 111$ \\
\hline 2005, ACT-2 & 0.321 & 0.246 & 0.405 & $42 / 131$ \\
\hline 2013, GEMINI 1 & 0.385 & 0.327 & 0.446 & $97 / 252$ \\
\hline 2017, OCTAVE & 0.404 & 0.348 & 0.462 & $113 / 280$ \\
\hline RBS 0 & 0.365 & 0.332 & 0.400 & $281 / 774$ \\
\hline 2005, ACT-1 & 0.148 & 0.076 & 0.269 & $8 / 54$ \\
\hline 2005, АCT-2 & 0.273 & 0.162 & 0.421 & $12 / 44$ \\
\hline 2013, GEMINI 1 & 0.354 & 0.284 & 0.432 & $56 / 158$ \\
\hline 2017, OCTAVE & 0.404 & 0.329 & 0.484 & $61 / 151$ \\
\hline RBS 0, SFS 0 & 0.345 & 0.300 & 0.394 & $137 / 407$ \\
\hline 2005, ACT-1 & 0.216 & 0.146 & 0.309 & $21 / 97$ \\
\hline 2005, АCT-2 & 0.360 & 0.272 & 0.458 & $36 / 100$ \\
\hline 2013, GEMINI 1 & 0.385 & 0.324 & 0.450 & $87 / 226$ \\
\hline 2017, OCTAVE & 0.412 & 0.353 & 0.474 & $103 / 250$ \\
\hline RBS 0 , SFS 0/1 & 0.371 & 0.335 & 0.409 & $247 / 673$ \\
\hline 2005, ACT-1 & 0.571 & 0.316 & 0.794 & $8 / 14$ \\
\hline 2005, ACT-2 & 0.194 & 0.090 & 0.369 & $6 / 31$ \\
\hline 2013, GEMINI 1 & 0.385 & 0.221 & 0.579 & $10 / 26$ \\
\hline 2017, OCTAVE & 0.333 & 0.190 & 0.516 & $10 / 30$ \\
\hline RBS 0 , SFS $2 / 3$ & 0.346 & 0.257 & 0.448 & $34 / 101$ \\
\hline 2005, ACT-1 & 0.095 & 0.024 & 0.311 & $2 / 21$ \\
\hline 2005, ACT-2 & 0.167 & 0.055 & 0.409 & $3 / 18$ \\
\hline 2013, GEMINI 1 & 0.222 & 0.086 & 0.465 & $4 / 18$ \\
\hline 2017, OCTAVE & 0.016 & 0.001 & 0.206 & $0 / 31$ \\
\hline RBS $2 / 3$, SFS $2 / 3$ & 0.145 & 0.078 & 0.254 & $9 / 88$ \\
\hline
\end{tabular}

Event rate and $95 \% \mathrm{Cl}$

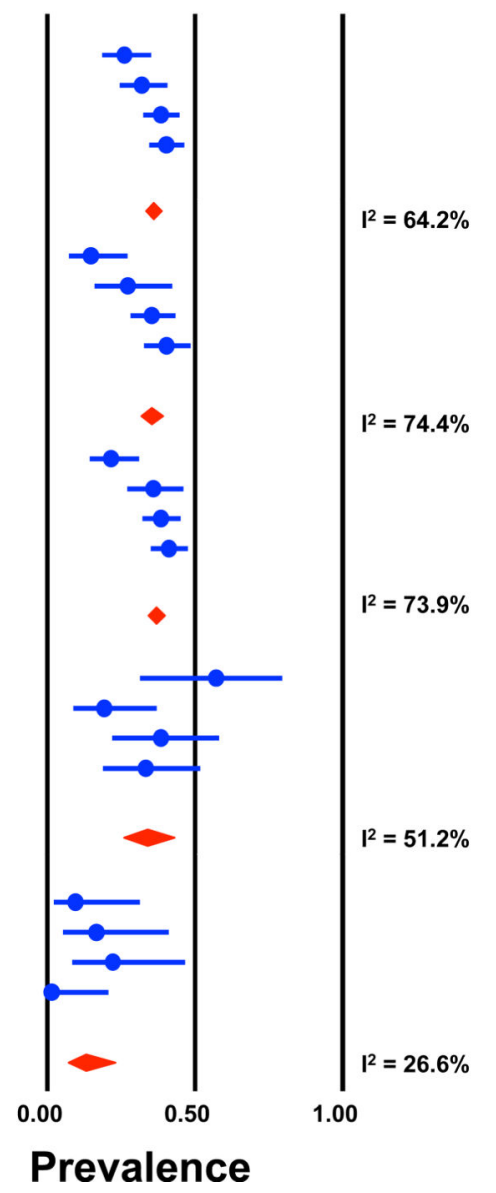

Figure 2: Pooled estimates from active intervention and placebo arms of ulcerative colitis clinical trials for prevalence of Mayo endoscopic scores during maintenance by patient reported outcome permutations

RBS: rectal bleedings core; SFS: stool frequency score; CI: Confidence interval Prevalence estimates are for the combined population of patients receiving active intervention (ie biologic or tofacitinib) or placebo. Individual study level estimates cannot be used to compare prevalence estimates across studies between different biologics or tofacitinib.

A: Endoscopic improvement (Mayo endoscopic score 0 or 1); B: Endoscopic remission (Mayo endoscopic scopre 0); C: Mild endoscopic activity (Mayo endoscopic score 1) 
Table 1:

Differences in prevalence of Mayo endoscopic sub-scores post-induction across patient reported outcome permutations when using local versus central endoscopy scores in tofacitinib trials

\begin{tabular}{|c|c|c|c|c|c|}
\hline \multirow[t]{2}{*}{ Patient-reported outcome } & \multirow[t]{2}{*}{ Endoscopic activity } & \multicolumn{2}{|c|}{ Local Reads } & \multicolumn{2}{|c|}{ Central Reads } \\
\hline & & $\mathbf{n} / \mathbf{N}$ & Prevalence & $\mathbf{n} / \mathbf{N}$ & Prevalence \\
\hline \multirow{3}{*}{$\mathrm{RBS} 0+\mathrm{SFS} 0 / 1$} & MES 0/1 $(\mathrm{p}<0.001)$ & $323 / 472$ & $68.4(64.1-72.5)$ & $242 / 472$ & $51.3(46.8-55.8)$ \\
\hline & MES $0(p=0.006)$ & $85 / 472$ & $18.0(14.8-21.7)$ & $55 / 472$ & $11.7(9.1-14.9)$ \\
\hline & $\operatorname{MES} 1(\mathrm{p}=0.001)$ & $238 / 472$ & $50.4(45.9-54.9)$ & $187 / 472$ & $39.6(35.3-44.1)$ \\
\hline \multirow{3}{*}{$\mathrm{RBS} 0+\mathrm{SFS} 0$} & MES 0/1 $(\mathrm{p}<0.001)$ & $150 / 196$ & $76.5(70.1-81.9)$ & $112 / 196$ & $57.1(50.1-63.9)$ \\
\hline & MES $0(p=0.09)$ & $43 / 196$ & $21.9(16.7-28.3)$ & $30 / 196$ & $15.3(10.9-21.0)$ \\
\hline & MES $1(p=0.01)$ & $107 / 196$ & $54.6(47.6-61.4)$ & $82 / 196$ & $41.8(35.1-48.9)$ \\
\hline \multirow{3}{*}{$\mathrm{RBS} 0+\mathrm{SFS} 2 / 3$} & $\operatorname{MES} 0 / 1(\mathrm{p}=0.32)$ & $35 / 154$ & $22.7(16.8-30.0)$ & $28 / 154$ & $18.2(12.9-25.1)$ \\
\hline & MES $0(p=0.79)$ & $7 / 154$ & $4.5(2.2-9.2)$ & $8 / 154$ & $5.2(2.6-10.0)$ \\
\hline & MES $1(p=0.21)$ & $28 / 154$ & $18.2(12.9-25.1)$ & $20 / 154$ & $13.0(8.5-19.3)$ \\
\hline \multirow{3}{*}{ RBS $2 / 3+\mathrm{SFS} 2 / 3$} & MES $0 / 1(p=0.46)$ & $10 / 140$ & $7.1(3.9-12.8)$ & $7 / 140$ & $5.0(2.4-10.1)$ \\
\hline & MES $0(p=1.00)$ & $0 / 140$ & $0.4(0.0-5.4)$ & $0 / 140$ & $0.4(0.0-5.4)$ \\
\hline & MES $1(\mathrm{p}=0.46)$ & $10 / 140$ & $7.1(3.9-12.8)$ & $7 / 140$ & $5.0(2.4-10.1)$ \\
\hline
\end{tabular}

RBS: rectal bleeding score; SFS: stool frequency score; MES: Mayo endoscopic score 
Table 2:

Differences in prevalence of Mayo endoscopic sub-scores during maintenance across patient reported outcome permutations when using local versus central endoscopy scores in tofacitinib trials

\begin{tabular}{|c|c|c|c|c|c|}
\hline \multirow{2}{*}{ Patient-reported outcome } & \multirow{2}{*}{ Endoscopic activity } & \multicolumn{2}{|c|}{ Local Reads } & \multicolumn{2}{|c|}{ Central Reads } \\
\hline & & $\mathbf{n} / \mathbf{N}$ & Prevalence & $\mathbf{n} / \mathbf{N}$ & Prevalence \\
\hline \multirow{3}{*}{$\mathrm{RBS} 0+\mathrm{SFS} 0 / 1$} & MES 0/1 $(\mathrm{p}<0.001)$ & $214 / 250$ & $85.6(80.7-89.4)$ & $179 / 250$ & $71.3(65.4-76.6)$ \\
\hline & MES $0(\mathrm{p}<0.001)$ & $111 / 250$ & $44.4(38.4-50.6)$ & $68 / 250$ & $27.1(22.0-32.9)$ \\
\hline & MES $1(p=0.49)$ & $61 / 151$ & $40.4(32.9-48.4)$ & $66 / 151$ & $43.7(36.0-51.7)$ \\
\hline \multirow{3}{*}{$\mathrm{RBS} 0+\mathrm{SFS} 0$} & MES 0/1 (p=0.001) & $135 / 151$ & $89.4(83.4-93.4)$ & $112 / 151$ & $74.2(66.6-80.5)$ \\
\hline & MES $0(\mathrm{p}=0.001)$ & $74 / 151$ & $49.0(41.1-56.9)$ & $46 / 151$ & $30.5(23.7-38.3)$ \\
\hline & MES $1(\mathrm{p}=0.56)$ & $103 / 250$ & $41.2(35.3-47.4)$ & $111 / 250$ & $44.2(38.2-50.4)$ \\
\hline \multirow{3}{*}{$\mathrm{RBS} 0+\mathrm{SFS} 2 / 3$} & $\operatorname{MES} 0 / 1(\mathrm{p}=0.57)$ & $10 / 30$ & $33.3(19.0-51.5)$ & $8 / 30$ & $26.7(13.9-45.0)$ \\
\hline & MES $0(\mathrm{p}=0.36)$ & $0 / 30$ & $1.6(0.1-21.1)$ & $2 / 30$ & $6.7(1.7-23.1)$ \\
\hline & MES $1(\mathrm{p}=0.25)$ & $10 / 30$ & $33.3(19.0-51.6)$ & $6 / 30$ & $20.0(9.3-37.9)$ \\
\hline \multirow{3}{*}{$\mathrm{RBS} 2 / 3+\mathrm{SFS} 2 / 3$} & $\operatorname{MES} 0 / 1(\mathrm{p}=1.00)$ & $0 / 31$ & $1.6(0.1-20.6)$ & $0 / 31$ & $1.6(0.1-20.6)$ \\
\hline & MES $0(p=1.00)$ & $0 / 31$ & $1.6(0.1-20.6)$ & $0 / 31$ & $1.6(0.1-20.6)$ \\
\hline & $\operatorname{MES} 1(\mathrm{p}=1.00)$ & $0 / 31$ & $1.6(0.1-20.6)$ & $0 / 31$ & $1.6(0.1-20.6)$ \\
\hline
\end{tabular}

RBS: rectal bleeding score; SFS: stool frequency score; MES: Mayo endoscopic score 